\title{
Trends in the treatment of cutaneous T-cell lymphoma - critical evaluation and perspectives on vorinostat
}

This article was published in the following Dove Press journal:

Blood and Lymphatic Cancer:Targets and Therapy

31 January 2012

Number of times this article has been viewed

\section{Sophia Rangwala \\ Madeleine Duvic \\ Chunlei Zhang}

Department of Dermatology, The University of Texas MD Anderson Cancer Center, Houston, Texas, USA
Correspondence: Chunlei Zhang Department of Dermatology, Box 1452 University of Texas MD Anderson Cancer Center, 1515 Holcombe Blvd, Houston, TX 77030, USA

Tel + I 7137454615

Fax + I 7137453597

Email czhang@mdanderson.org
Abstract: Epigenetic modification with small molecule histone deacetylase inhibitors has been a promising new anti-neoplastic approach for various solid and hematological malignancies, particularly cutaneous T-cell lymphoma (CTCL). Oral vorinostat was the first histone deacetylase inhibitor approved to enter the clinical oncology market for treating CTCL patients who have progressive, persistent, or recurrent disease after failing two systemic therapies. In two phase II clinical trials, oral vorinostat was found to be safe and effective at a dose of $400 \mathrm{mg} / \mathrm{day}$, with an overall response rate of $24 \%-30 \%$ in heavily pretreated patients with advanced CTCL, including those with large-cell transformed mycosis fungoides and Sézary syndrome. About half of CTCL patients receiving vorinostat also experienced substantial relief in pruritus and thus a marked improvement in quality of life. A subsequent follow-up study reported long-term safety and clinical benefits of vorinostat in patients with refractory CTCL, regardless of previous treatment failures. The most frequent side effects of vorinostat include gastrointestinal symptoms, fatigue, and thrombocytopenia. These adverse reactions are dose-related and reversible upon cessation of therapy. Preclinical studies have supported the therapeutic potential of vorinostat by demonstrating in vitro and in vivo anti-tumor activities against CTCL, including selective induction of apoptosis in malignant $\mathrm{T}$ cells, inhibition of angiogenesis, suppression of signal transducer and activator of transcription proteins, and up-regulation of pro-apoptotic proteins. Identification of biomarkers of response and resistance will help select CTCL patients most likely to benefit from treatment and guide the design of effective combination therapies.

Keywords: cutaneous T-cell lymphoma, mycosis fungoides, Sézary syndrome, histone deacetylase inhibitors, vorinostat

\section{Cutaneous T-cell lymphoma}

Cutaneous T-cell lymphoma (CTCL) is a heterogeneous group of extranodal nonHodgkin's lymphomas that is characterized by clonal proliferation of mature $\mathrm{T}$ cells that localize to the skin. Mycosis fungoides (MF), the most common subtype of CTCL, represents a predominance of mature effector memory T cells in the skin. ${ }^{1}$ Clinically, it often presents as indolent and pruritic skin lesions that usually begin as patches or plaques, and may progress to become tumors or erythroderma (covering $\geq 80 \%$ body surface area). ${ }^{2}$ Sézary syndrome is an aggressive leukemic variant of CTCL that can arise de novo or evolve from long-standing MF. Sézary syndrome patients present with a triad of generalized erythroderma, lymphadenopathy, and malignant central memory $T$ cells in the peripheral blood ( $>1000$ malignant T cells $/ \mu \mathrm{L})$ known as Sézary cells. ${ }^{1-3}$ CTCL is generally considered advanced when the patient has erythroderma, tumors, or nodal involvement (stage IIB or higher). We have previously shown that 
the median overall survival in patients with erythrodermic CTCL is 5.1 years, with the strongest predictive factors being elevated serum lactate dehydrogenase, advanced age, and the absolute Sézary cell count. ${ }^{4}$

The association of CTCL with increased class II HLA-DR5 and $D Q B^{*} 03$ alleles suggests that the disease may arise in the setting of aberrant antigen stimulation or a genetic predisposition as seen with other autoimmune skin diseases. ${ }^{5}$ Mutations in $\mathrm{T}$ cells may promote both $\mathrm{T}$ cell accumulation via defects in the Fas/Fas ligand death pathway ${ }^{6}$ and attraction to the skin via mutations in skin-homing molecules. ${ }^{3}$ Although a number of infectious and environmental triggers have been hypothesized, studies have failed to identify a consistent antigen stimulant. ${ }^{78}$ Additionally, Pautrier's microabscesses, a clustering of malignant $\mathrm{CD} 4^{+} \mathrm{T}$ cells around Langerhans cells, may be observed in the epidermis of MF lesions. These epidermal dendritic cells are thought to present antigens and activate $\mathrm{T}$ cells to clonally expand, ${ }^{9,10}$ and may thereby explain the presence of both tumor cells and an inflammatory infiltrate in early MF lesions. Malignant T cells in Sézary syndrome typically have a $\mathrm{CD} 4^{+} \mathrm{CD} 26^{-}$phenotype and exhibit dysfunctional apoptotic mechanisms, such as loss of Fas and expression of bcl-2, which result in the loss of activation-induced cell death and prolonged life-span. ${ }^{11}$ Progression of disease is marked by the dominance of a Th2 cytokine profile among tumor cells and the loss of $\mathrm{CD} 8^{+}$cytotoxic $\mathrm{T}$ cells, thus further depressing cellular immunity and allowing the accumulation of malignant clones. ${ }^{12}$

\section{Current treatment options}

Food and Drug Administration (FDA)-approved therapeutic modalities for CTCL are limited. At this time, the options include topical and oral bexarotene, intravenous denileukin diftitox, photopheresis, oral vorinostat, and intravenous depsipeptide. ${ }^{2,13-15}$ Many non-approved agents, such as topical steroids, topical mustargen, phototherapy, interferons, and chemotherapy, are commonly used as a standard of care and may be more effective than approved therapies. ${ }^{2}$ Despite the treatment options available, CTCL rarely undergoes complete remission and no specific agent has been correlated with improved survival. Refractory or transformed disease is often associated with poor prognosis. ${ }^{2}$

A combination of topical therapies and systemic biological response modifiers is often used for patients refractory to first-line skin-directed treatments who have less than $10 \%$ involvement of body surface area (stage IA), for patients with more than $10 \%$ involvement (stage IB), or for those with lymphadenopathy (stage IIA). The most common combinations include phototherapy plus retinoids (bexarotene, acitretin, or isotretinoin) or interferon (IFN), or mustargen plus topical steroids. Total body skin electron beam radiation is suggested for those who fail to respond to other therapies and need palliation due to extensive skin involvement. This therapy should be followed by long-term maintenance therapy, such as mustargen, phototherapy, oral bexarotene, or if the patient is young and healthy, an allogeneic stem cell transplant. ${ }^{16}$

Patients with advanced CTCL may respond to localized radiation, denileukin diftitox, or histone deacetylase (HDAC) inhibitors (HDAC-Is). ${ }^{17}$ Chemotherapies, including nucleoside analogues (gemcitabine, pentostatin) or pegylated liposomal doxorubicin, are reserved for patients with refractory tumors or nodal disease. ${ }^{17}$ Although combined chemotherapies are often effective for a short duration, they subject the patient to further immunosuppression, leading to line-induced sepsis and other opportunistic infections. Because patients can experience considerable morbidity from progression of disease and cumulative adverse effects from multiple treatments, development of novel therapies with better therapeutic indices and more durable remissions is critical. HDAC-Is have been especially effective for various hematological malignancies, most particularly CTCL. This is evidenced by the recent FDA approval of the HDAC-Is vorinostat in 2006 and romidepsin in 2009 for the treatment of relapsed or refractory CTCL. ${ }^{14,15,18}$ Ongoing clinical trials with these and several other HDAC-Is demonstrate the clinical potential of this novel targeted therapy towards the management of advanced CTCL.

\section{The mechanistic profile of HDAC-Is}

Epigenetic modifications involve changes in gene or protein expression not secondary to direct DNA mutations. Examples are microRNA silencing, DNA methylation, and DNA histone acetylation. The addition and removal of acetyl groups to positively charged lysine tails of core nucleosomal histones play an important role in transcriptional regulation. This is a dynamic process of chromatin remodeling that depends on the opposing activities of histone acetyltransferases and HDACs. ${ }^{19}$ Histone acetylation by histone acetyltransferases unfolds chromatin by neutralizing the lysine tails, thereby reducing the affinity of the histones to the negatively charged DNA phosphate backbone. Acetylation thus promotes binding of transcription factor complexes to facilitate gene transcription. In contrast, histone deacetylation by HDACs condenses chromatin and thereby prohibits transcription. HDAC-Is, by reversibly or irreversibly blocking the active sites of HDACs, allow the unopposed acetylation of histones 
and other non-histone proteins. These small molecule agents are relevant to cancer therapeutics because they may restore the expression of tumor suppressor genes that are abnormally repressed in malignant cells, such as those involved in cell cycle arrest, differentiation, apoptosis, and angiogenesis. ${ }^{20}$

In humans, 18 HDACs have been identified and can be subdivided into five groups (Table 1): class I (HDAC1, HDAC2, HDAC3, HDAC8), class IIa (HDAC4, HDAC5, HDAC7, HDAC9), class IIb (HDAC6, HDAC10), class III (SIRT 1-7), and class IV (HDAC11). ${ }^{19}$ Class I, II, and IV enzymes are zinc-dependent, while class III enzymes are nicotinamide adenine dinucleotide (NAD)-dependent enzymes. Class I HDACs primarily localize to the nucleus and are small, expressed ubiquitously, and generally involved in cell proliferation and differentiation. Class II HDACs are larger, act in association with tissue-specific transcription factors, and have both histone and non-histone targets. Class IIa enzymes are shuttled to and from the nucleus, whereas class IIb enzymes primarily localize to the cytoplasm. Class III HDACs are homologues of yeast $\operatorname{Sir} 2$ and are insensitive to inhibition by class I and II HDAC-Is due to a non-homologous catalytic site. Because the primarily nuclear HDAC11 is homologous to both class I and II HDACs, it has been categorized separately as a class IV enzyme. Knocking out individual HDAC isotypes in mouse models has demonstrated the physiological importance of these enzymes. Interestingly, deleting class I HDACs is lethal in the embryonic and perinatal stages, while deleting class II HDACs usually produces viable mice with significant developmental defects. ${ }^{21}$

Aberrant overexpression, function, and recruitment of class I and II HDACs are associated with malignancies including T-cell lymphomas. ${ }^{19,22}$ Of note, increased expression of particularly class I HDAC1, HDAC2, and HDAC3 has been shown to be essential for cancer cell proliferation and survival. ${ }^{23}$ Additionally, class I HDAC expression in both solid and hematological malignancies tends to correlate with a worse prognosis, ${ }^{23}$ with high expression of HDAC2 being seen with more aggressive forms of CTCL. ${ }^{22}$ Alternatively, the expression of Class II HDACs 4, 5, 6, 7, and 10 tends to be associated with a better prognosis, especially in CTCL (HDAC6) and non-small cell lung cancer. ${ }^{22,23}$ The reasons for these correlations are not yet understood, but nevertheless suggest that selective inhibition of class I HDACs may be a more effective therapeutic strategy.

HDAC-Is have demonstrated anti-tumor activity in solid and hematological malignancies, particularly in T-cell lymphomas and most remarkably in CTCL. The rationale for the anti-tumor activity of HDAC-I involves a complicated and poorly understood range of pleiotropic effects. Gene expression profiling of T-cell lymphoblastic cell lines incubated with HDAC-Is vorinostat and romidepsin demonstrated up to $22 \%$ of the genome being affected within 16 hours of treatment. ${ }^{24}$ In several tumors and cell lines, HDAC-I treatment has been shown to induce accumulation of acetylated histones as well as the acetylation of non-histone protein substrates, such as transcriptional co-activators (RB, MSL-3), transcription factors (eg, p53, c-myc, E2F, bcl-6, GATA, NF- $\kappa B$, CREB, IRF), signaling mediators (STAT-3, IRS-1), steroid receptors (eg, androgen, estrogen, glucocorticoid), DNA repair enzymes (eg, KU70, FEN1, WRN), cytoskeletal components (eg, $\alpha$-tubulin), molecular chaperones (eg, heat shock protein-90), and nuclear import factors. ${ }^{24-26}$ Unregulated acetylation of these proteins leads to alterations in transcription, mitosis, and protein stability, with downstream effects on tumor proliferation, survival, and maintenance.

HDAC-Is can repress tumor proliferation by inducing cell cycle arrest at the G1/S and G2/M time points. By repressing cyclin D and cyclin A and up-regulating p27, p16, and particularly $\mathrm{p} 21$, vorinostat and romidepsin reduce activity of CDK4 and CDK2 and interrupt cell cycle progression at the G1/S checkpoint. ${ }^{24,26-28}$ Also, for reasons that are still elusive, HDAC-Is preferentially activate intrinsic and extrinsic apoptosis in malignant cells at low concentrations for which healthy cells are relatively resistant..$^{29-31}$ The intrinsic pathway involves mitochondrial permeabilization

Table I Human histone deacetylases (HDACs)

\begin{tabular}{llllll}
\hline Class & Class I & Class IIa & Class IIb & Class III & Class IV \\
\hline Members & HDACI, 2, 3, and 8 & HDAC4, 5, 7, and 9 & HDAC6, I0 & SIRTI-7 & HDACII \\
MW & 22-25 KD & I20-I35 KD & I20-I35 KD & $40-50$ KD & I20-I35 KD \\
Localization & & & & & + \\
$\quad$ Nuclear & + & + & + & + & + \\
$\quad$ Cytoplasmic & & + & + & + & Zn $^{++}$ \\
Binding site & Zn & Zn & Zn & \\
Homology with yeast & RPD3 deacetylase & HDAl deacetylase & HDAI deacetylase & SIRT2 family & HDAI deacetylase \\
\hline
\end{tabular}

Reprinted with permission from US Oncology, 2009;5(I):27-31. 
in response to internal cellular triggers. This in turn induces release of cytochrome $\mathrm{c}$, which activates the caspase cascade via caspase 9. HDAC-Is affect the intrinsic pathway via repression of anti-apoptotic proteins (bcl-2, bcl-xL, XIAP) and activation of pro-apoptotic proteins (bim, bax). In fact, over-expression of bcl-2 and occasionally bcl-xL has been shown to protect lymphoma cells from panobinostat-induced death. ${ }^{32,33}$ The extrinsic pathway is activated by receptors responding to extracellular stimuli. The subsequent induction of the downstream death-inducing signaling complex (DISC) activates the caspase cascade via caspase 8 . HDAC-Is, particularly vorinostat, have been shown to increase gene expression for both death receptors (eg, Fas, TRAIL receptors DR4/DR5) and their ligands, decrease expression of extrinsic pathway inhibitors (eg, c-FLIP, cIAP2, X1 AP), and increase DISC activity. ${ }^{34}$ Interestingly, data also suggest sensitization of leukemia cells to TRAIL-induced apoptosis likely results from inhibition of class I versus class II HDACs. ${ }^{35}$ Moreover, HDAC-I induction of autophagy has been consistently demonstrated, and was first suggested when panobinostat and vorinostat-treated cancer cells were found to undergo cell death despite inhibition of caspase-mediated apoptotic cascades. ${ }^{32,36,37}$

In addition to disrupting cell cycle and survival pathways, HDAC-Is also appear to inhibit angiogenesis by up-regulating anti-angiogenic proteins (eg, thrombospondin-1, VonHippel-Landau factor, neurofibromin-2) and down-regulating pro-angiogenic proteins (eg, vascular endothelial growth factor [VEGF], hypoxia-induced protein [HIF]-1 $\alpha) .{ }^{14,38,39}$ Finally, HDAC-Is may repress inflammation via inhibition of pro-inflammatory cytokines and up-regulation of antiinflammatory cytokines, and its use in inflammatory and autoimmune conditions is currently being investigated. ${ }^{40}$

Many structurally variable subclasses of HDAC-Is have been studied, including hydroxamic acids, cyclic peptides, benzamides, short-chain fatty acids, and electrophilic ketones. ${ }^{26}$ These HDAC-Is can either act as pan-inhibitors which target zinc-containing HDAC isoforms of at least classes I and II, or selective inhibitors which target particular isoforms or subclasses of HDACs. However, there is so far no data that indicates inhibiting a particular HDAC isoform is correlated with increased efficacy, or that selective HDAC-Is are correlated with decreased adverse events.

The biological basis for why CTCL is especially sensitive to HDAC-Is remains uncertain. Several HDAC-Is are currently undergoing extensive clinical evaluation in CTCL. ${ }^{20}$ In addition to the FDA-approved agents vorinostat and romidepsin, ${ }^{14,15,18}$ panobinostat (LBH589) and belinostat
(PXD101) have achieved partial and complete responses in CTCL patients with refractory disease ${ }^{41,42}$ Herein, we present preclinical and clinical data supporting the use of vorinostat for the treatment of advanced CTCL.

\section{Vorinostat}

Vorinostat (suberoylanilide hydroxamic acid, SAHA, Zolinza $\left.^{\circledR}\right)$ is an orally administered HDAC-I ( $\mathrm{t}_{1 / 2}=2$ hours) approved for treatment of CTCL in October 2006. This small molecule has an empirical formula of $\mathrm{C}_{14} \mathrm{H}_{20} \mathrm{~N}_{2} \mathrm{O}_{3}$ and a molecular weight of $264.32 \mathrm{~g} / \mathrm{mol}$. It reversibly inhibits class I HDACs and class IIb HDAC6, and weakly inhibits class IIa HDACs. ${ }^{43,44}$ We discuss the mechanistic understanding of vorinostat in CTCL below and then describe the pivotal CTCL trials that led to its FDA approval.

\section{Preclinical studies}

\section{Selective induction of apoptosis}

Vorinostat has been shown to inhibit the cell viability of several malignant cell lines and human tumor xenografts in mice at concentrations to which normal cells stay relatively unaffected. ${ }^{45}$ We have previously demonstrated that clinically relevant doses $(1-2 \mu \mathrm{M})$ of vorinostat induce apoptosis in both CTCL cell lines and primary malignant $\mathrm{T}$ cells, but not normal $\mathrm{T}$ cells from healthy donors. ${ }^{14,29}$ However, this difference in sensitivity to vorinostat-induced apoptosis is unlikely secondary to differing levels of HDAC inhibition because both tumor and normal cells had a similar accumulation of acetylated histones. Rather, transformed cells treated with vorinostat may be exclusively sensitive to reactive oxygen species accumulation and caspase activation, ${ }^{46}$ or may be unable to recover from vorinostat-induced DNA double-strand breaks. ${ }^{47}$ Further studies are needed to better understand these processes. ${ }^{31}$

\section{Accumulation of acetylated histones}

Histone acetylation after HDAC-I treatment has been shown to correlate with drug activity in both normal and transformed cells. ${ }^{26}$ For this reason, acetylated histones have been measured as an intermediary marker in phase I clinical trials, such as with vorinostat. ${ }^{48}$ Vorinostat results in increased protein levels of acetylated histones (H2B, H3, and H4) in both sensitive and resistant CTCL cell lines in vitro. ${ }^{29}$ Furthermore, MF lesions had unexpectedly high H4 acetylation in keratinocyte and $\mathrm{T}$ cell nuclei at baseline, the significance of which is unknown. ${ }^{14}$ Because vorinostat treatment significantly decreased the lymphocyte infiltrate in 
MF tissue, the effect of this HDAC-I on histone acetylation was difficult to assess. Based on this data, it seems that the degree of histone acetylation is not predictive of response in CTCL.

\section{Up-regulation of the intrinsic apoptosis pathway}

The cyclin-dependent kinase inhibitor p21 (WAF1/CIP1) is one of the most commonly reported genes to be up-regulated by vorinostat and other HDAC-Is, ${ }^{29,49}$ and results in downstream induction of G2 cell cycle arrest and apoptosis. Although up-regulation of p21 occurred in response to vorinostat in CTCL cell lines, immunoblot analysis showed that this effect was independent of the tumor suppressor p53. ${ }^{29}$ In addition, the balance between protein expression of the anti-apoptotic bcl-2 and the pro-apoptotic bax is critical in controlling the activation of caspases by regulating the release of cytochrome c from mitochondria. ${ }^{50}$ Of interest, vorinostat treatment of three CTCL cell lines and patientderived primary Sézary cells in vitro did not change bcl-2, but increased bax, activated caspase-3, and cleaved poly (ADP-ribose) polymerase. ${ }^{31}$

\section{Modulation of STAT signaling}

CTCL cells have been shown to constitutively express signal transducer and activator of transcription (STAT) proteins, which dimerize and become phosphorylated after growth factor stimulation to induce gene transcription and promote cell proliferation. ${ }^{51,52}$ Vorinostat did not decrease protein expression of STAT-3 and phospho-STAT-3, but did decrease STAT-6 and phospho-STAT-6 in CTCL cell lines and primary Sézary cells. ${ }^{29}$ Interestingly, following vorinostat treatment, the distribution of phospho-STAT-3 protein changed from a predominantly nuclear to cytoplasmic location in skin lesions of responders. ${ }^{14}$ Nuclear accumulation of STAT-1 and high levels of nuclear phospho-STAT-3 were present in malignant T cells in CTCL skin lesions, and correlated with a lack of clinical response to vorinostat. ${ }^{52}$ Thus, deregulation of STAT activity is likely to play a key role in vorinostat response and resistance in CTCL.

\section{Cytokine modulation}

Vorinostat may also have immunomodulatory effects, by shifting the immune milieu away from the Th2-dominant profile of malignant CTCL cells. A recent study found selective down-regulation of the Th2 cytokine interleukin (IL)-10 likely mediated by the STAT3 pathway. ${ }^{53}$ This effect was seen to a lesser extent in the expression of IL-4. Additionally, vorinostat enhanced expression of the Th1 cytokine IFN- $\gamma$ and mildly decreased expression of the T-cell growth-stimulating cytokine IL-2.

\section{Inhibition of angiogenesis}

Angiogenesis is a key process during tumor development and metastasis that is tightly controlled by the balance between positive and negative environmental signals that induce and inhibit angiogenesis, respectively. ${ }^{54}$ We found that treatment with vorinostat decreases the expression of the pro-angiogenic protein VEGF in CTCL. ${ }^{14}$ In addition, thrombospondin-1, a potent inhibitor of angiogenesis, was increased 8-fold in a cDNA microarray analysis of vorinostat-treated HH CTCL cell. ${ }^{14}$ We confirmed this finding by demonstrating that in CTCL patients' paired skin lesions, treatment with vorinostat ( 2 hours, 4 weeks, and 8 weeks) often induced a significant decrease in microvascular density and an increase in dermal thrombospondin-1 in responders, when compared to baseline. ${ }^{14}$ Thus, inhibition of angiogenesis and up-regulation of thrombospondin-1 by vorinostat may participate in inhibiting CTCL growth.

\section{Potential biomarkers}

HR23B, a candidate biomarker identified in a genome-wide loss-of-function screen for HDAC-I-induced apoptosis, has an important role in shuttling ubiquitinated cargo proteins to the proteasome. ${ }^{55}$ Interestingly, HR23B controls the sensitivity of CTCL cells to vorinostat. ${ }^{56}$ Moreover, proteasome activity is deregulated in vorinostat-treated CTCL cells through a mechanism dependent on HR23B, and vorinostat sensitizes CTCL cells to the effects of the proteasome inhibitor bortezomib. Notably an analysis of HR23B levels in CTCL skin lesions taken from our phase II trial of vorinostat ${ }^{14}$ demonstrated a positive correlation between HR23B expression and clinical efficacy of vorinostat. ${ }^{56}$

\section{Pharmacokinetics}

Pharmacokinetic studies have shown the plasma level of vorinostat and the orally administered dose (200-600 mg) to be linearly related. ${ }^{48}$ Although the mean $\mathrm{C}_{\max }$ after oral vorinostat (range: $304-658 \mathrm{ng} / \mathrm{mL}$ ) was less than intravenous vorinostat at an orally equivalent dose (range: 1088-2306 ng/ $\mathrm{mL})$, the mean apparent half-life $\left(\mathrm{t}_{1 / 2}\right)$ of oral vorinostat (range: 91.6-127 minutes) was longer than that of the intravenous form (range: $34.7-42.4$ minutes). While the $\mathrm{C}_{\max }$ proportionally increases with increasing dose of vorinostat in CTCL lines and primary Sézary cells to be the $t_{1 / 2}$, the median time to reach $\mathrm{C}_{\max }\left(\mathrm{t}_{\max }\right)$, and the apparent clearance $\left(\mathrm{CL}_{\text {app }}\right)$ remain constant. Oral vorinostat has reasonably 
good absorption, with a fasting bioavailability of $43 \%$ at doses of 200 and $400 \mathrm{mg}$. Of note, the rate and extent of absorption is similar when taken in fasting versus nonfasting states, although administration with food may help relieve gastrointestinal discomfort associated with the drug.

\section{Clinical studies of oral vorinostat for refractory CTCL}

\section{Phase I trial}

Oral vorinostat at a dose of $400 \mathrm{mg}$ /day has a rapid onset of action, and improved the nodes, blood burden, and itching in heavily pretreated patients. ${ }^{14,18} \mathrm{~A}$ phase I trial of patients with advanced cancer first noted the clinical efficacy of oral vorinostat for a patient with refractory CTCL who had failed five systemic regimens. ${ }^{48}$ The disease stabilized when $200 \mathrm{mg}$ oral vorinostat was given twice daily for 4 months.

\section{Phase Ila dose-ranging trial}

A single-center phase II dose-ranging study for oral vorinostat was conducted by our group in 33 patients with refractory or relapsed CTCL who had been unresponsive to a median of five systemic therapies (range: $1-15) .{ }^{14}$ Of the enrolled patients, $85 \%$ had advanced disease (stage IIB or higher) and $33 \%$ had SS. The first cohort received $400 \mathrm{mg}$ daily of vorinostat, the second received $300 \mathrm{mg}$ twice daily for
3 days with a rest of 4 days, and the third received $300 \mathrm{mg}$ twice daily for 14 days with a rest of 7 days followed by $200 \mathrm{mg}$ twice daily. Demographics, such as age and gender, were similar among these three groups. The clinical response for each cohort and suggested dose modification schedule are summarized in Table 2. The first cohort (400 mg daily) had the best response without the significant doselimiting thrombocytopenia seen in the third cohort (300 mg twice daily).

The primary end point of the study was to assess the complete response (CR) and partial response (PR) rates. Secondary objectives were to evaluate the time to progressive disease, response duration, relief of pruritus, and safety. The response to therapy was categorized according to the Physician's Global Assessment for CR, PR, stable disease, or progressive disease. ${ }^{14}$ Skin involvement was assessed as the percent body surface area of patch, plaque, or tumor disease. Per intent-to-treat analysis, eight of 33 (24\%) patients achieved a documented PR with no CRs. An additional 14 (42\%) patients experienced improvement in pruritus, such that a total of 19 of 33 (58\%) enrolled patients clinically benefited from treatment. Vorinostat was clinically effective for a variety of CTCL phenotypes, including patients with early stage refractory MF, advanced tumors with histologic largecell transformation, and nodal and/or blood involvement.

Table 2 Phase II data on oral vorinostat in patients with cutaneous T-cell lymphoma

\begin{tabular}{|c|c|c|c|}
\hline & \multicolumn{3}{|c|}{ Treatment group } \\
\hline & Group I & Group 2 & Group 3 \\
\hline Dosing schedule & $400 \mathrm{mg} \mathrm{qd}^{*}$ & $\begin{array}{l}300 \mathrm{mg} \text { bid } \times 3 \text { days } / \mathrm{wk} \times 4 \mathrm{wk} \text {, } \\
\text { then } 5 \text { days } / \mathrm{wk}\end{array}$ & $\begin{array}{l}300 \mathrm{mg} \text { bid } \times 14 \text { days with } 7 \text { days } \\
\text { rest maintenance: } 200 \mathrm{mg} \text { bid }\end{array}$ \\
\hline \multicolumn{4}{|l|}{ Dose modification } \\
\hline Ist dose reduction & $350 \mathrm{mg}$ qd & $250 \mathrm{mg} \times 3$ days $/ w k$ & $200 \mathrm{mg}$ bid \\
\hline 2 nd dose reduction & $300 \mathrm{mg}$ qd & $200 \mathrm{mg}$ bid $\times 3$ days $/$ wk & $200 \mathrm{mg}$ bid $\times 5$ days $/$ wk \\
\hline \multicolumn{4}{|l|}{ Clinical response } \\
\hline Partial response (PR) & $31 \%(4 / 13)$ & $9 \%(I / I I)$ & $33 \%(3 / 9)$ \\
\hline Median PR duration (range/weeks) & $15(8-24)$ & $16(8-24)$ & $13(3-2 \mid)$ \\
\hline Mean time to PR (weeks) & II & 4 & II \\
\hline LN regression (at 4 weeks) & $78 \%(7 / 9)$ & $38 \%(3 / 8)$ & $56 \%(5 / 9)$ \\
\hline$>50 \% \downarrow$ pruritus & $82 \%(9 / 11)$ & $67 \%(8 / 12)$ & $50 \%(4 / 8)$ \\
\hline \multicolumn{4}{|l|}{ Adverse events (Grade 3/4) } \\
\hline Thrombocytopenia & $8 \%(1 / 13)$ & $8 \%(1 / 12)$ & $42 \%(5 / 12)$ \\
\hline Anemia & $8 \%(1 / 13)$ & $17 \%(2 / 12)$ & $0 \%(0 / 0)$ \\
\hline Deep vein thrombosis & $0 \%(0 / 0)$ & $25 \%(3 / 12)$ & $0 \%(0 / 0)$ \\
\hline Dehydration & $8 \%(1 / 13)$ & $0 \%(0 / 0)$ & $17 \%(2 / 12)$ \\
\hline Pyrexia & $0 \%(0 / 0)$ & $25 \%(3 / 12)$ & $0 \%(0 / 0)$ \\
\hline Hypotension & $0 \%(0 / 0)$ & $17 \%(2 / 12)$ & $0 \%(0 / 0)$ \\
\hline Pulmonary embolism & $0 \%(0 / 0)$ & $17 \%(2 / 12)$ & $0 \%(0 / 0)$ \\
\hline Sepsis & $0 \%(0 / 0)$ & $8 \%(1 / 12)$ & $8 \%(I / I 2)$ \\
\hline
\end{tabular}

Note: *Initial three patients treated with vorinostat $250 \mathrm{mg} / \mathrm{m}^{2} /$ day.

Information extracted and adapted from Duvic, et al. Blood. 2007;109:31-39. Reprinted with permission from US Oncology, 2009;5(I):27-3I. 
Notably, lymphadenopathy, when present, improved in responders as well. The response rate in patients who had received prior oral bexarotene $(23 \%)$ or not $(27 \%)$ was similar. The median time to response was 11.9 weeks, and the median duration of response was 15.1 weeks (range: 9.4-19.4 weeks). The median duration of response was lowest in the second cohort, who received intermittent dosing (9.4 weeks), and highest in the first cohort, who were continuously treated with $400 \mathrm{mg}$ daily (16.1 weeks). Overall, oral vorinostat $400 \mathrm{mg}$ daily provided the most favorable risk-benefit profile, and the dose of $400 \mathrm{mg}$ /day was selected for evaluation in a pivotal open-label, multi-center phase IIb trial. ${ }^{18}$

\section{Phase Ilb single-arm trial}

A registration phase IIb study of oral vorinostat at $400 \mathrm{mg}$ daily enrolled 74 patients with stage IB-IVA MF/SS. ${ }^{18}$ Two dose modifications were allowed: $300 \mathrm{mg}$ daily or $300 \mathrm{mg}$ daily for 5 days per week. Safety assessments were performed with the National Cancer Institute Common Terminology Criteria for Adverse Events version 3.0. Inclusion criteria were patients with MF/SS stage IB-IVB who had progressive, persistent, or recurrent disease that was resistant to at least two prior systemic treatments, one of which being bexarotene. Patients with an Eastern Cooperative Oncology Group (ECOG) performance status of $0-2$ and adequate hematologic, hepatic, and renal function were included. Patients with prior use of HDAC-Is or anticancer treatments within 3 weeks of study entry were excluded. Unlike the previous phase II study, skin involvement was assessed with the modified severity weighted assessment tool (mSWAT), which weighted plaque and tumor disease more heavily. Of the $82 \%$ of patients who had refractory advanced stage MF/SS ( $\geq$ stage IIB), about $30 \%$ achieved an overall response. The median time to response was less than 2 months. Median response duration and time to progression in advanced-stage responders were not reached, but were estimated to be more than 6.1 and 9.8 months, respectively. Median time to progression in all patients was 4.9 months. Pruritus relief was observed in $32 \%$ of study patients, including $25 \%$ of those who had not met the objective criteria for a cutaneous response.

\section{Continuation phase trial}

Of the 74 patients who participated in the phase IIb trial, a continuation phase study was implemented for six patients who had received vorinostat for at least 2 years. ${ }^{57}$ Upon enrollment, five of the six patients had achieved an objective response, with one patient with facial tumors having experienced a durable $\mathrm{CR}$, and one patient having prolonged stable disease. Of these six patients, one patient discontinued treatment because of a serious adverse event and three additional patients discontinued because of progressive disease (day 780, day 1008, and day 1066). One patient discontinued vorinostat (day 803) after relocating, and subsequently continued treatment with vorinostat by prescription. One patient remained on vorinostat therapy.

\section{Safety profile}

Oral vorinostat is generally well tolerated, with side effects being dose-related and reversible upon cessation of therapy. As is often noted with other HDAC-Is, the most common toxicities with oral vorinostat in these studies were fatigue and gastrointestinal symptoms, including diarrhea, dysgeusia, nausea, and dehydration from the diarrhea and/or decreased appetite (Table 2). In the Phase IIa study, one serious grade $3 / 4$ adverse experiences included thrombocytopenia (19\%), dehydration $(8 \%)$, vomiting $(8 \%)$, anemia $(8 \%)$, deep vein thrombosis $(8 \%)$, pyrexia $(8 \%)$, hypotension $(5 \%)$, pulmonary embolism $(5 \%)$, and sepsis $(5 \%)$. All of these events resolved, except in 2 patients whose dehydration/vomiting persisted. ${ }^{14}$ Grade 3/4 thrombocytopenia was most common in the third cohort $(42 \%)$, who received the higher dose of $300 \mathrm{mg}$ twice daily for 14 days, when compared to the other cohorts $(8 \%)$. Of note, thrombocytopenia was reversible 4-5 days after treatment cessation. ${ }^{58}$ In the Phase $\mathrm{IIb}$ dose-ranging study, vorinostat at $400 \mathrm{mg}$ daily was generally tolerated well, with less than $15 \%$ of patients requiring dose reductions due to intolerability. ${ }^{18}$ The most common adverse events, most of which were grade 2 or less, included gastrointestinal symptoms (diarrhea [49\%], nausea [43\%], anorexia [26\%], dysgeusia, dry mouth, vomiting, constipation, and anorexia), fatigue (46\%), thrombocytopenia (22\%), weight loss, alopecia, muscle spasms, creatinine elevation, anemia, and chills. Grade 3/4 adverse events involved fatigue $(5 \%)$, deep venous thrombosis/pulmonary embolism (5\%), thrombocytopenia (5\%), and nausea (4\%). Three patients died during the course of the study from disease progression, ischemic stroke, and an unexplained cause.

Since five patients suffered pulmonary embolisms while on these vorinostat trials, there was concern as to whether HDAC-I had pro-coagulant properties; however, researchers now believe the events to be likely coincidental and more directly related to the pro-coagulability of relapsed cancers, especially SS. None of the patients in these vorinostat trials experienced grade 3/4 neutropenia. QTc prolongation was initially a concern with HDAC-I use, particularly romidepsin, although no clinically concerning 
cardiac sequelae has been seen with either HDAC-I. In the phase IIb study, grade 1/2 QTc prolongation was reported in only three patients, and was not correlated with any cardiac toxicity. Still, the prescribing practitioner should be aware of the possible risk, and should consider monitoring for low $\mathrm{K}^{+}$and $\mathrm{Mg}^{+}$ions prior to administration and avoiding treatment of patients who have a history of arrhythmias or are on medications known to prolong QTc intervals.

\section{Potential combination treatments}

Because long-term remissions are rare in advanced or otherwise refractory CTCL, vorinostat is administered as a third-line monotherapy until the patient experiences disease progression or medication intolerance. Unfortunately, only about one-third of refractory CTCL patients respond to vorinostat and these patients can experience a limited response duration. The development of synergistic combination treatments may be more effective for overcoming HDAC-I resistance by promoting increased and more durable response rates. Combined regimens may also allow for lower doses of each individual agent to be used, thereby decreasing the risk of side effects without compromising on efficacy.

Preclinical and clinical studies for several solid and hematologic malignancies have shown targeted cytotoxic agents such as anthracyclines ${ }^{59,60}$ and all-trans retinoic acid, ${ }^{61}$ antiangiogenesis agents, radiation, ${ }^{62}$ and other anticancer treatments to enhance the anti-tumor effects of HDAC-Is. ${ }^{26}$ For advanced CTCL, a phase I dose-ranging trial of vorinostat and bexarotene combination therapy was recently conducted. ${ }^{63}$ The maximum tolerated dose was established to be lower than each drug individually: vorinostat at $200 \mathrm{mg}$ daily and bexarotene at $300 \mathrm{mg} / \mathrm{m}^{2}$ daily. Of the 22 patients evaluated, 4 (18\%) had an objective response, and 7 (32\%) had relief of pruritus. The most common side effects have been hypothyroidism $(35 \%)$, fatigue $(30 \%)$, and hypertriglyceridemia $(30 \%)$.
Additionally, several case reports suggest that vorinostat has better clinical efficacy in CTCL when combined with bexarotene plus fenofibrate, ${ }^{64}$ IFN- $\gamma,{ }^{65}$ or IFN- $\alpha$ plus extracorporeal photopheresis. ${ }^{66}$ Preclinical studies in T-cell lymphomas have also increased interest in assessing other combination therapies in CTCL patients. For instance, vorinostat has demonstrated synergy with the proteasome inhibitor bortezomib in cells derived from CTCL, adult T-cell leukemia/ lymphoma, and B-cell leukemia/lymphoma patients. ${ }^{26,67,68}$ In CTCL cells, the combination up-regulates p21 and p27, and activates p38 mitogen-activated protein kinase, an enzyme responsive to stress stimuli. ${ }^{67}$ Notably, HDAC6 is a class II HDAC that has been shown to have a pro-survival role via promotion of both aggresomes and autophagosomes. Inhibition of HDAC6 via vorinostat may in turn prevent bortezomib-induced aggresome production. ${ }^{69}$ Vorinostat and other HDAC-Is can also exert synergistic or additive anti-tumor effects in CTCL when combined with a bcl-2/ bcl-xL antagonist, ${ }^{70}$ JAK/STAT inhibitor, ${ }^{52}$ phosphoinositide-3 kinase (PI3K) inhibitor, ${ }^{71}$ or PIM kinase inhibitors. ${ }^{71}$ These potential combinations are listed in Table 3 and merit further evaluation.

\section{Future perspective}

Both the clinical efficacy and the minimally immunosuppressive nature of vorinostat compared to traditional chemotherapy make this small molecule appealing for cancer treatment, particularly for CTCL as immunosuppression is severe in advanced patients. Despite the significant and sustainable single-agent activity of vorinostat in refractory advancedstage CTCL, clinical hurdles include the need to increase the response rate and address the frequent development of HDAC-I resistance. Since we have only begun to understand the biological mechanisms by which vorinostat selectively targets tumor cells, the hope is that additional studies can

Table 3 Combination studies of vorinostat in CTCL

\begin{tabular}{|c|c|c|c|}
\hline Combination & Model & Activity & Genes/mechanisms \\
\hline Vorinostat + Bexarotene & Phase I trial (22 CTCL patients) & 4/22 OR, 7/22 pruritus $\downarrow$ & Regulation of RXR/RAR ${ }^{63}$ \\
\hline Vorinostat + Bexarotene + Fenofibrate & Case report (I MF patient) & $\mathrm{CR}$ & Regulation of co-repressors? ${ }^{64}$ \\
\hline Vorinostat + INF $\gamma$ & Case report (3 SS patients) & $3 \mathrm{OR}$ & T-reg/TGF- $\beta \downarrow ?^{65}$ \\
\hline Vorinostat $+I N F \alpha+E C P$ & Case report (3 MF/SS patients) & $2 \mathrm{OR}, \mathrm{I} \mathrm{SD}$ & T-reg/TGF- $\beta \downarrow ?^{66}$ \\
\hline Vorinostat + JAK inhibitor & CTCL cell lines & Synergistic & $\begin{array}{l}\text { p-STAT3/bcl-2/bcl-xL/survivin/mcl- } \mid \downarrow \\
\text { c-Myc/OSM } \downarrow^{52}\end{array}$ \\
\hline Vorinostat + Bortezomib & CTCL cell lines & Synergistic & Proteasome activity $\downarrow p 21 / p 27 / p-p 38 \uparrow 67$ \\
\hline Vorinostat $+\mathrm{PI} 3 \mathrm{~K}$ inhibitor & CTCL cell lines & Synergistic & GSK3ß $\uparrow^{71}$ \\
\hline Vorinostat + PIM kinase inhibitor & CTCL cell lines & Additive & $\mathrm{PIMI} / \mathrm{PIM} 2 \uparrow 71$ \\
\hline Vorinostat + HSP90 inhibitor & CTCL cell lines & Antagonistic & HSP90 $\downarrow 71$ \\
\hline
\end{tabular}

Abbreviations: $C T C L$, cutaneous T-cell lymphoma; OR, overall response; $C R$, complete response; $S D$, stable disease; $R X R / R A R$, retinoid receptors; PI3K, phosphoinositide-3 kinase; T-reg, regulatory T cells; TGF- $\beta$, transforming growth factor- $\beta$. 
help identify reliable markers of response and resistance that will allow the personalized selection of patients most likely to benefit from treatment. Further, numerous preclinical and clinical studies have shown vorinostat to not only be effective as monotherapy, but also capable of potentiating the antitumor effects of other cancer therapeutics. This has spurred the ongoing clinical investigation of a number of combination therapies, which we hope will maximize the clinical potential of this novel agent.

\section{Acknowledgments}

Dr Duvic received clinical and basic support from Aton/ Merck for conducting clinical trials. Dr Zhang received research support from Merck and Co Inc, Dermatology Foundation, Ladies Leukemia League Inc, and Novartis Pharm. This article was independently commissioned and no fee was received for preparation of the manuscript.

\section{Disclosure}

The authors declare no conflicts of interest in this report.

\section{References}

1. Campbell JJ, Clark RA, Watanabe R, Kupper TS. Sezary syndrome and mycosis fungoides arise from distinct T-cell subsets: a biologic rationale for their distinct clinical behaviors. Blood. 2010;116(5):767-771.

2. Duvic M, Foss FM. Mycosis fungoides: pathophysiology and emerging therapies. Semin Oncol. 2007;34(6 Suppl 5):S21-S28.

3. Olsen EA, Rook AH, Zic J, et al. Sezary syndrome: immunopathogenesis, literature review of therapeutic options, and recommendations for therapy by the United States Cutaneous Lymphoma Consortium (USCLC). J Am Acad Dermatol. 2011;64(2):352-404.

4. Vidulich KA, Talpur R, Bassett RL, Duvic M. Overall survival in erythrodermic cutaneous T-cell lymphoma: an analysis of prognostic factors in a cohort of patients with erythrodermic cutaneous T-cell lymphoma. Int J Dermatol. 2009;48(3):243-252.

5. Jackow CM, McHam JB, Friss A, Alvear J, Reveille JR, Duvic M. HLA-DR5 and DQB $1 * 03$ class II alleles are associated with cutaneous T-cell lymphoma. J Invest Dermatol. 1996;107(3):373-376.

6. Ni X, Zhang C, Talpur R, Duvic M. Resistance to activation-induced cell death and bystander cytotoxicity via the Fas/Fas ligand pathway are implicated in the pathogenesis of cutaneous $\mathrm{T}$ cell lymphomas. J Invest Dermatol. 2005;124(4):741-750.

7. Whittemore AS, Holly EA, Lee IM, et al. Mycosis fungoides in relation to environmental exposures and immune response: a case-control study. J Natl Cancer Inst. 1989;81(20):1560-1567.

8. Mirvish ED, Pomerantz RG, Geskin LJ. Infectious agents in cutaneous T-cell lymphoma. J Am Acad Dermatol. 2011;64(2):423-431.

9. Vega F, Luthra R, Medeiros LJ, et al. Clonal heterogeneity in mycosis fungoides and its relationship to clinical course. Blood. 2002;100(9): 3369-3373.

10. Berger CL, Hanlon D, Kanada D, et al. The growth of cutaneous T-cell lymphoma is stimulated by immature dendritic cells. Blood. 2002;99(8):2929-2939.

11. Kacinski BM, Flick M. Apoptosis and cutaneous T cell lymphoma. Ann NY Acad Sci. 2001;941:194-199.

12. Kim EJ, Hess S, Richardson SK, et al. Immunopathogenesis and therapy of cutaneous T cell lymphoma. J Clin Invest. 2005;115(4): 798-812.
13. Duvic M, Hymes K, Heald P, et al. Bexarotene is effective and safe for treatment of refractory advanced-stage cutaneous T-cell lymphoma: multinational phase II-III trial results. J Clin Oncol. 2001;19(9):2456-2471.

14. Duvic M, Talpur R, Ni X, et al. Phase 2 trial of oral vorinostat (suberoylanilide hydroxamic acid, SAHA) for refractory cutaneous T-cell lymphoma (CTCL). Blood. 2007;109(1):31-39.

15. Piekarz RL, Frye R, Turner M, et al. Phase II multi-institutional trial of the histone deacetylase inhibitor romidepsin as monotherapy for patients with cutaneous T-cell lymphoma. J Clin Oncol. 2009;27(32):5410-5417.

16. Duvic M, Donato M, Dabaja B, et al. Total skin electron beam and non-myeloablative allogeneic hematopoietic stem-cell transplantation in advanced mycosis fungoides and Sezary syndrome. J Clin Oncol. 2010;28(14):2365-2372.

17. Duvic M. Systemic monotherapy vs combination therapy for CTCL: rationale and future strategies. Oncology (Williston Park). 2007; 21(2 Suppl 1):33-40.

18. Olsen EA, Kim YH, Kuzel TM, et al. Phase IIb multicenter trial of vorinostat in patients with persistent, progressive, or treatment refractory cutaneous T-cell lymphoma. J Clin Oncol. 2007;25(21): 3109-3115.

19. Beumer JH, Tawbi H. Role of histone deacetylases and their inhibitors in cancer biology and treatment. Curr Clin Pharmacol. 2010;5(3):196-208.

20. Ellis L, Pili R. Histone Deacetylase Inhibitors: Advancing Therapeutic Strategies in Hematological and Solid Malignancies. Pharmaceuticals (Basel). 2010;3(8):2411-2469.

21. Lemoine M, Younes A. Histone deacetylase inhibitors in the treatment of lymphoma. Discov Med. 2010;10(54):462-470.

22. Marquard L, Gjerdrum LM, Christensen IJ, Jensen PB, Sehested M, Ralfkiaer E. Prognostic significance of the therapeutic targets histone deacetylase 1, 2, 6 and acetylated histone $\mathrm{H} 4$ in cutaneous T-cell lymphoma. Histopathology. 2008;53(3):267-277.

23. Weichert W. HDAC expression and clinical prognosis in human malignancies. Cancer Lett. 2009;280(2):168-176.

24. Peart MJ, Smyth GK, van Laar RK, et al. Identification and functional significance of genes regulated by structurally different histone deacetylase inhibitors. Proc Natl Acad Sci U S A. 2005;102(10): 3697-3702.

25. Dokmanovic M, Clarke C, Marks PA. Histone deacetylase inhibitors: overview and perspectives. Mol Cancer Res. 2007;5(10):981-989.

26. Zain J, O'Connor OA. Targeting histone deacetyalses in the treatment of B- and T-cell malignancies. Invest New Drugs. 2010;28 Suppl 1(1): S58-S78.

27. Sambucetti LC, Fischer DD, Zabludoff S, et al. Histone deacetylase inhibition selectively alters the activity and expression of cell cycle proteins leading to specific chromatin acetylation and antiproliferative effects. J Biol Chem. 1999;274(49):34940-34947.

28. Sandor V, Senderowicz A, Mertins S, et al. P21-dependent g(1) arrest with downregulation of cyclin D1 and upregulation of cyclin $\mathrm{E}$ by the histone deacetylase inhibitor FR901228. Br J Cancer. 2000;83(6): $817-825$.

29. Zhang C, Richon V, Ni X, Talpur R, Duvic M. Selective induction of apoptosis by histone deacetylase inhibitor SAHA in cutaneous T-cell lymphoma cells: relevance to mechanism of therapeutic action. J Invest Dermatol. 2005;125(5):1045-1052.

30. Emanuele S, Lauricella M, Tesoriere G. Histone deacetylase inhibitors: apoptotic effects and clinical implications (Review). Int J Oncol. 2008;33(4):637-646.

31. Nalabothula N, Carrier F. Cancer cells' epigenetic composition and predisposition to histone deacetylase inhibitor sensitization. Epigenomics. 2011;3(2):145-155.

32. Ellis L, Bots M, Lindemann RK, et al. The histone deacetylase inhibitors LAQ824 and LBH589 do not require death receptor signaling or a functional apoptosome to mediate tumor cell death or therapeutic efficacy. Blood. 2009;114(2):380-393. 
33. Shao W, Growney JD, Feng Y, et al. Activity of deacetylase inhibitor panobinostat (LBH589) in cutaneous T-cell lymphoma models: Defining molecular mechanisms of resistance. Int $J$ Cancer. 2010;127(9):2199-2208.

34. Droin N, Guery L, Benikhlef N, Solary E. Targeting apoptosis proteins in hematological malignancies. Cancer Lett. 2011;30:30.

35. Inoue $\mathrm{S}$, Mai A, Dyer MJ, Cohen GM. Inhibition of histone deacetylase class I but not class II is critical for the sensitization of leukemic cells to tumor necrosis factor-related apoptosis-inducing ligand-induced apoptosis. Cancer Res. 2006;66(13):6785-6792.

36. Shao Y, Gao Z, Marks PA, Jiang X. Apoptotic and autophagic cell death induced by histone deacetylase inhibitors. Proc Natl Acad Sci U S A. 2004;101(52):18030-18035.

37. Robert T, Vanoli F, Chiolo I, et al. HDACs link the DNA damage response, processing of double-strand breaks and autophagy. Nature. 2011;471(7336):74-79.

38. Kwon HJ, Kim MS, Kim MJ, Nakajima H, Kim KW. Histone deacetylase inhibitor FK228 inhibits tumor angiogenesis. Int $J$ Cancer. 2002;97(3):290-296.

39. Qian DZ, Kato Y, Shabbeer S, et al. Targeting tumor angiogenesis with histone deacetylase inhibitors: the hydroxamic acid derivative LBH589. Clin Cancer Res. 2006;12(2):634-642.

40. Grabiec AM, Tak PP, Reedquist KA. Function of histone deacetylase inhibitors in inflammation. Crit Rev Immunol. 2011;31(3):233-263.

41. Duvic M, Vanaclocha F, Bernengo MG, et al. Phase II study of oral panobinostat (LBH589), a potent pan-deacetylase inhibitor, in patients with refractory Cutaneous T-cell Lymphoma (CTCL) (abstract). J Clin Oncol. 2008;26(May 20 Suppl):8555.

42. Pohlman B, Advani R, Duvic M, et al. Final Results of a Phase II Trial of Belinostat (PXD101) in Patients with Recurrent or Refractory Peripheral or Cutaneous T-Cell Lymphoma (abstract). Blood. 2009;110:3453.

43. Kelly WK, Marks PA. Drug insight: Histone deacetylase inhibitors development of the new targeted anticancer agent suberoylanilide hydroxamic acid. Nat Clin Pract Oncol. 2005;2(3):150-157.

44. Bradner JE, West N, Grachan ML, et al. Chemical phylogenetics of histone deacetylases. Nat Chem Biol. 2010;6(3):238-243.

45. Lane AA, Chabner BA. Histone deacetylase inhibitors in cancer therapy. $J$ Clin Oncol. 2009;27(32):5459-5468.

46. Ruefli AA, Ausserlechner MJ, Bernhard D, et al. The histone deacetylase inhibitor and chemotherapeutic agent suberoylanilide hydroxamic acid (SAHA) induces a cell-death pathway characterized by cleavage of Bid and production of reactive oxygen species. Proc Natl Acad Sci U S A. 2001;98(19):10833-10838.

47. Lee JH, Choy ML, Ngo L, Foster SS, Marks PA. Histone deacetylase inhibitor induces DNA damage, which normal but not transformed cells can repair. Proc Natl Acad Sci U S A. 2010;107(33):14639-14644.

48. Kelly WK, O'Connor OA, Krug LM, et al. Phase I study of an oral histone deacetylase inhibitor, suberoylanilide hydroxamic acid, in patients with advanced cancer. J Clin Oncol. 2005;23(17):3923-3931.

49. Gui CY, Ngo L, Xu WS, Richon VM, Marks PA. Histone deacetylase (HDAC) inhibitor activation of p21 WAF1 involves changes in promoter-associated proteins, including HDAC1. Proc Natl Acad Sci US A. 2004;101(5):1241-1246.

50. Tait SW, Green DR. Mitochondria and cell death: outer membrane permeabilization and beyond. Nat Rev Mol Cell Biol. 2010;11(9): $621-632$.

51. Qin JZ, Zhang CL, Kamarashev J, Dummer R, Burg G, Dobbeling U. Interleukin-7 and interleukin-15 regulate the expression of the bcl-2 and c-myb genes in cutaneous T-cell lymphoma cells. Blood. 2001;98(9):2778-2783.

52. Fantin VR, Loboda A, Paweletz CP, et al. Constitutive activation of signal transducers and activators of transcription predicts vorinostat resistance in cutaneous T-cell lymphoma. Cancer Res. 2008;68(10):3785-3794.

53. Tiffon C, Adams J, van der Fits L, et al. The histone deacetylase inhibitors vorinostat and romidepsin downmodulate IL-10 expression in cutaneous T-cell lymphoma cells. Br J Pharmacol. 2011;162(7):1590-1602.
54. Cao Y. Molecular mechanisms and therapeutic development of angiogenesis inhibitors. Adv Cancer Res. 2008;100:113-131.

55. Fotheringham S, Epping MT, Stimson L, et al. Genome-wide lossof-function screen reveals an important role for the proteasome in HDAC inhibitor-induced apoptosis. Cancer Cell. 2009;15(1):57-66.

56. Khan O, Fotheringham S, Wood V, et al. HR23B is a biomarker for tumor sensitivity to HDAC inhibitor-based therapy. Proc Natl Acad Sci U S A. 2010;107(14):6532-6537.

57. Duvic M, Olsen EA, Breneman D, et al. Evaluation of the longterm tolerability and clinical benefit of vorinostat in patients with advanced cutaneous T-cell lymphoma. Clin Lymphoma Myeloma. 2009;9(6):412-416.

58. O’Connor OA, Heaney ML, Schwartz L, et al. Clinical experience with intravenous and oral formulations of the novel histone deacetylase inhibitor suberoylanilide hydroxamic acid in patients with advanced hematologic malignancies. J Clin Oncol. 2006;24(1):166-173.

59. Cheriyath V, Kuhns MA, Kalaycio ME, Borden EC. Potentiation of apoptosis by histone deacetylase inhibitors and doxorubicin combination: cytoplasmic cathepsin B as a mediator of apoptosis in multiple myeloma. Br J Cancer. 2011;104(6):957-967.

60. Terpos E. The synergistic effect of panobinostat (LBH589) with melphalan or doxorubicin on multiple myeloma cells; rationale for the use of combination regimens in myeloma patients. Leuk Res. 2011;35(3): 295-296.

61. Bellos F, Mahlknecht U. Valproic acid and all-trans retinoic acid: meta-analysis of a palliative treatment regimen in AML and MDS patients. Onkologie. 2008;31(11):629-633.

62. Page P, Yang LX. Novel chemoradiosensitizers for cancer therapy. Anticancer Res. 2010;30(9):3675-3682.

63. Dummer R, Hymes K, Sterry W, et al. Vorinostat in combination with bexarotene in advanced cutaneous T-cell lymphoma: A phase I study (abstract). J Clin Oncol. 2009;27(15s):8572.

64. Steinhoff M, Beyer M, Roewert-Huber J, et al. Complete clinical remission of tumor-stage mycosis fungoides after acute extensive skin necroses, granulomatous reaction, and fever under treatment with bexarotene, vorinostat, and high-dose fenofibrate. J Am Acad Dermatol. 2008;58(5 Suppl 1):S88-S91.

65. Gardner JM, Introcaso CE, Nasta SD, Kim EJ, Vittorio CC, Rook AH. A novel regimen of vorinostat with interferon gamma for refractory Sezary syndrome. J Am Acad Dermatol. 2009;61(1):112-116.

66. Sanli H, Akay BN, Anadolu R, Ozcan M, Saral S, Akyol A. The efficacy of vorinostat in combination with interferon alpha and extracorporeal photopheresis in late stage mycosis fungoides and Sezary syndrome. J Drugs Dermatol. 2011;10(4):403-408.

67. Heider U, Rademacher J, Lamottke B, et al. Synergistic interaction of the histone deacetylase inhibitor SAHA with the proteasome inhibitor bortezomib in cutaneous T cell lymphoma. Eur J Haematol. 2009;82(6):440-449.

68. Zhang QL, Wang L, Zhang YW, et al. The proteasome inhibitor bortezomib interacts synergistically with the histone deacetylase inhibitor suberoylanilide hydroxamic acid to induce T-leukemia/lymphoma cells apoptosis. Leukemia. 2009;23(8):1507-1514.

69. Truee O, Matthias P. Interplay between histone deacetylases and autophagy - from cancer therapy to neurodegeneration. Immunol Cell Biol. 2011;29(10):103.

70. Chen J, Fiskus W, Eaton K, et al. Cotreatment with BCL-2 antagonist sensitizes cutaneous T-cell lymphoma to lethal action of HDAC7Nur77-based mechanism. Blood. 2009;113(17):4038-4048.

71. Wozniak MB, Villuendas R, Bischoff JR, et al. Vorinostat interferes with the signaling transduction pathway of T-cell receptor and synergizes with phosphoinositide-3 kinase inhibitors in cutaneous T-cell lymphoma. Haematologica. 2010;95(4):613-621. 
Blood and Lymphatic Cancer: Targets and Therapy

Dovepress

\section{Publish your work in this journal}

Blood and Lymphatic Cancer: Targets and Therapy is an international, peer-reviewed, open access journal focusing on blood and lymphatic cancer research, identification of therapeutic targets and the optimal use of preventative and integrated treatment interventions to achieve improved outcomes, enhanced survival and quality of life for the

cancer patient. The manuscript management system is completely online and includes a very quick and fair peer-review system. Visit http://www.dovepress.com/testimonials.php to read real quotes from published authors.

Submit your manuscript here: http://www.dovepress.com/blood-and-lymphatic-cancer-targets-and-therapy-journal 